\title{
Future

\section{A novel antimicrobial approach based on the inhibition of zinc uptake in Salmonella enterica}

In this review we discuss evidences suggesting that bacterial zinc homeostasis represents a promising target for new antimicrobial strategies. The ability of the gut pathogen Salmonella enterica sv Typhimurium to withstand the host responses aimed at controlling growth of the pathogen critically depends on the zinc importer ZnuABC. Strains lacking a functional ZnuABC or its soluble component ZnuA display a dramatic loss of pathogenicity, due to a reduced ability to express virulence factors; withstand the inflammatory response; and compete with other gut microbes. Based on this data, ZnuA was chosen as a candidate for the rational design of novel antibiotics. Through a combination of structural and functional investigations, we have provided a proof of concept of the potential of this approach.

First draft submitted: 22 February 2017; Accepted for publication: 3 April 2017; Published online: 21 June 2017

Keywords: Salmonella enterica • zinc homeostasis $\bullet$ ZnuA x-ray structures

The discovery of novel antimicrobials is not keeping pace with the diffusion of antibiotic resistance in pathogenic bacteria $[1,2]$. This situation represents a major challenge for maintaining the fundamental ability to control bacterial-caused diseases that has contributed significantly to improve human health in the last century. Current antibiotics hinder a limited number of metabolic processes (primarily membrane integrity, cell wall and nucleic acid synthesis and mRNA translation). Hence, significant efforts have been made to identify other metabolic pathways which might represent effective targets for new antimicrobials.

An approach in this direction is to carry out systematic analyses of the contribution of all the genes of a microorganism to its ability to infect an animal host. Interesting drug target candidates may be identified in those genes associated to loss of pathogenicity in infection studies though not essential for bacterial viability under laboratory growth conditions. These analyses can be conveniently integrated by studies aimed at the identification of genes that are upregulated during animal infections. The gut pathogen Salmonella enterica sv Typhimurium (S. Typhimurium), that causes self-limiting gastroenteritis in humans but brings about lethal systemic infections in mice, can be considered a model of choice for this kind of experiments and for the identification of antimicrobial targets in Gram-negative bacteria [3,4]. This approach, however, has led to the worrying suggestion that limited possibilities to develop novel classes of antibiotics exists, as the most essential genes belong to pathways already targeted by available antibiotics [5].

Another option is to look for less selective targets. In this regard, an interesting possibility is to exploit strategies that interfere with metal homeostasis in bacteria. For example, strategies to target iron metabolism include the development of siderophore-antibiotic conjugates acting as Trojan horses [6,7] or the use of different gallium formulations [8]. However, the high redundancy of the iron
Andrea Battistoni', Serena Ammendola ${ }^{1,2}$, Emilia Chiancone $^{3}$ \& Andrea Ilari ${ }^{*, 4}$ 'Dipartimento di Biologia, Università di Roma Tor Vergata, Via della Ricerca Scientifica snc, 00133 Rome, Italy ${ }^{2}$ CTIF, Center for TelelnFrastructures, Università di Roma Tor Vergata, Via della Ricerca Scientifica snc, 00133 Rome Italy ${ }^{3}$ Dipartimento di Scienze Biochimiche, Università Sapienza di Roma, P.le Aldo Moro 5, 00185 Rome, Italy ${ }^{4}$ Consiglio Nazionale delle Ricerche (CNR)-Istituto di Biologia e Patologia Molecolari (IBPM) c/o Dipartimento di Scienze Biochimiche, Università Sapienza di Roma, P.le Aldo Moro 5, 00185 Rome, Italy

*Author for correspondence:

Tel.: +3906 49910910

Fax: +39064440062

andrea.ilari@uniroma1.it 
importing strategies in pathogenic bacteria renders the development of resistance possible. As an alternative, we are exploring the possibility to identify molecules able to interfere with zinc homeostasis. Zinc is the cofactor of more than $5 \%$ of all bacterial proteins and several of them play key roles in bacterial metabolism. Moreover, since several zinc proteins contribute to the management of gene expression, a reduction in metal uptake may significantly modulate the composition of the bacterial proteome thereby leading to changes in the expression and activity of zinc-containing proteins as well as of proteins which do not use this cofactor [9]. Our studies on $S$. Typhimurium confirmed the critical importance of an efficient zinc import for the ability of this pathogen to infect the host.

\section{Proteins contributing to zinc uptake in Salmonella}

The outer membrane of Salmonella is thought to be permeable to low MW hydrophilic molecules and to small ions [10]. In agreement with the suggestion that zinc ions passively diffuse from the extracellular space into the periplasm, no specific outer membrane receptors involved in zinc uptake have been identified in this microorganism. In contrast, the transport of zinc through the inner membrane is mediated by a small set of specific metal transporters.

The intracellular supply of this metal is primarily ensured by the low-affinity importer ZupT, an integral inner membrane protein that belongs to the ZIP (ZRT, IRT-like protein) family [11]. As demonstrated by studies on E. coli [12], ZupT has the capability to mediate the import of a broad range of divalent metals, with a preference for zinc ions. While in E. coli, ZupT is expressed constitutively in $S$. Typhimurium, ZupT expression slightly increases under zinc-limiting conditions [13]. ZupT activity is driven by the proton-motive force and disruption of the phage shock protein system Psp reduces Salmonella ability to import zinc and limits its growth in macrophages, by failing to compete with the phagosomal divalent ion pump, Nramp1 [14]. Deletion of ZupT causes changes in intracellular zinc concentration that leads to small growth defects in media devoid of zinc and an increased sensitivity to oxidative stress. These phenotypes, however, are enhanced significantly in a Salmonella strain unable to acquire zinc through the high-affinity zinc uptake system ZnuABC [13].

ZnuABC is a zinc-specific metal importer present in most Gram-negative bacteria, which ensures metal import under conditions of zinc shortage. In Salmonella and other enterobacteria, such as E. coli and Campylobacter jejuni, this is the only transporter ensuring efficient zinc uptake in metal-poor environments.
It is a high affinity ATP-binding cassette-type transporter formed by three proteins: a soluble component, ZnuA, that captures $\mathrm{Zn}$ (II) in the periplasmic compartment, a transmembrane channel, ZnuB, which allows zinc transport through the inner membrane and the cytosolic ATPase component, ZnuC, which provides the energy necessary for ion transport through the inner membrane [15]. In addition, Salmonella encodes for an auxiliary soluble periplasmic component of the ZnuABC system, named ZinT, which contributes to zinc recruitment in the periplasm by interacting with ZnuA [16,17].

Both the znuABC operon and the zinT gene are regulated by Zur, a zinc-binding metalloprotein that coordinates the expression of a set of genes required for the adaptation of bacteria to conditions of zinc paucity. When saturated with zinc, Zur binds specific DNA motifs located in promoter regions and downregulates transcription. In contrast, when intracellular zinc levels are not sufficient to ensure its complete metallation, Zur loses its affinity for its target DNA sequences, thereby allowing expression of the controlled genes. Interestingly, zinT and $z n u A$ are transcriptionally activated at different zinc concentrations [16], suggesting that subtle differences in the Zur-binding sites reflect a hierarchical role of Zur-regulated genes in the response to zinc starvation, useful to establish a dynamic response to changes in zinc availability [18,19]. Despite the expression of zupT increases under condition of zinc deficiency, this gene is not regulated by Zur even though a putative Zur-binding site sequence has been identified in its promoter [13].

\section{Efficient zinc ion import ability is critical for Salmonella pathogenicity}

Deletion of the $z n u A B C$ operon does not alter Salmonella ability to grow in zinc-rich media. In contrast, Salmonella strains lacking the whole znuABC operon, or just the gene encoding for the soluble component ZnuA, display an evident growth delay compared with the wild-type strain when cultivated in media with low zinc content (chemically defined media or rich media supplemented with metal chelators). Even though the growth rate of a $z u p T$ mutant in zincpoor media is similar to that of a wild-type strain, the double mutant lacking both $z n u A$ and $z u p T$ is severely compromised, confirming that the corresponding proteins are the two major zinc importers in Salmonella and that $\mathrm{ZnuABC}$ is the most important zinc importer under condition of severe zinc deficiency $[9,13]$.

Even more noteworthy is the behavior of these mutants in infection models. The lethal dose of znuABC Salmonella strains is several orders of magnitude higher than that of wild-type strains, either when 
mice are infected orally or when animals are infected via the intraperitoneal route [20]. This finding indicates that zinc availability inside the host is limited both in the gastrointestinal tract and in systemic sites and that $\mathrm{ZnuABC}$ is indispensable to guarantee an adequate zinc import. In support of this view, ZnuA has been shown to be highly expressed in intracellular $S$. Typhimurium recovered from infected cells, in other words, macrophages and epithelial-like cells, and from the spleens of infected mice. The level of ZnuA accumulation in intracellular bacteria is comparable to that observed in bacteria cultured in a zinc-devoid medium, even though the apparent zinc concentration in eukaryotic cells is close to $0.2 \mathrm{mM}$ [20]. This observation suggests that $Z$ nuABC is required to have access to the very tiny quota of intracellular zinc that is not tightly bound to proteins.

ZnuABC has also a critical role in the ability of $S$. Typhimurium to colonize the gut. In fact, Salmonellainduced intestinal inflammation produces a response characterized by a marked neutrophil recruitment. In turn, as a strategy to contain pathogen proliferation, neutrophils release large amounts of calprotectin, an antimicrobial protein able to chelate zinc and manganese, to starve microbes of these essential metals [21]. However, ZnuABC mediates resistance to such an antimicrobial strategy and promotes Salmonella growth in the inflamed gut by conferring a growth advantage over resident competing microbes. As a matter of fact, wild-type and znuABC Salmonella strains interact differently with the intestinal microbiota and induce a markedly different effect on the composition of the gut flora [22].

\section{Consequences of the inhibition of ZnuABC on bacterial metabolism}

Analyses of Salmonella responses to zinc shortage have evidenced that inactivation of the ZnuABC transporter determines major ionomic and proteomic intracellular changes. The intracellular metal content of wild-type and znuA Salmonella strains grown in a chemically defined medium has been analyzed by inductively coupled plasma-mass spectrometry [23], revealing unexpected zinc-dependent alterations in the content of different elements, such as magnesium, calcium and iron. At the same time, the addition of zinc to the growth medium proved to reduce significantly the intracellular content of manganese and copper of the znuA strain. Moreover, inactivation of the ZnuABC system increases $S$. Typhimurium susceptibility to cadmium exposure, a phenotype that can be reverted by zinc supplementation [16,24]. These observations suggest that disruption of the zinc importing ability interferes with homeostasis of many other metals, thereby expanding the repertoire of metabolic pathways that could be affected by shortage of this metal.

A label-free shotgun proteomic analysis has shown major proteome alterations between wild-type and the znuA mutant strain under zinc shortage [23]. Proteins that are differentially expressed mainly belong to pathways related to protein and amino acids biosynthesis, glycolysis, TCA (tricarboxylic acid cycle) and transport of anions and sugars. In particular, there is an upregulation of nonzinc requiring ribosomal proteins, a strategy already described in Bacillus subtilis to ensure the correct metal placement in essential zinc-cofactored enzymes [25]. In addition, several virulence-related proteins are downregulated in the znuA mutant grown under zinc stringency, as well as proteins necessary for Salmonella motility. In agreement with these data, a recent study has highlighted that the inactivation of the znuABC system leads to a significant reduction of swimming motility on agar plates that lack zinc, due to a global transcriptional repression of the flagellar regulon, and a reduced ability to form biofilms in zinc-devoid liquid cultures. Interestingly, an enhancement of the secretion of autoinducer-2, the signaling molecule involved in intercellular communication, has been also observed, thereby suggesting that zinc availability influences the transition from the planktonic to the biofilm lifestyle [26].

Overall, the above findings collectively indicate that the inactivation of ZnuABC-mediated zinc import leads to broad metabolic changes that are associated to a dramatic reduction of pathogenicity. An additional feature that marks $\mathrm{ZnuABC}$ as an interesting target for antimicrobials is that bacterial strains without a functional transporter maintain the ability to develop a mild and short-lasting infection that elicits a cell-mediated immune response which protects mice against challenge infections with virulent $S$. Typhimurium strains [27]. The efficacy of the $z n u A B C$ strain as a live attenuated vaccine has been documented both in systemic infection and in the gastrointestinal model on streptomycin pretreated mice [28] and in pigs [28-30].

\section{The ZnuABC transporter in Salmonella enterica}

The above-mentioned studies indicate that ZnuABC plays a central role in Salmonella pathogenicity and strongly suggest that this transporter, or merely its soluble periplasmic component $\mathrm{ZnuA}$, represents putative targets to find new drugs against Gram-negative bacteria. The latter possibility is supported either by the observation that mammalians do not express proteins showing significant similarity with ZnuA and ZnuB or by a thorough knowledge of the structural properties of the periplasmic component $\mathrm{ZnuA}$. ZnuA belongs to 
a group of periplasmic solute binding proteins (PBPs) named cluster 9 including manganese-specific PBPs. The proteins belonging to this family are formed by two $(\alpha / \beta)_{4}$ sandwich domains connected by a long $\alpha$-helix. Each domain is composed by a parallel fourstranded $\beta$-sheet with strand sequence $2-3-1-4$ and four $\alpha$-helices, two on each side of the $\beta$-sheet, that alternate with the $\beta$-strands in the primary sequence. The metal-binding site is placed at the interface between the two domains and the metal is coordinated tetrahedrally by residues belonging to both the domains. Mn-specific PBPs coordinate the metal via two histidines and two acidic residues (MntC and PsaA) [31,32] or three histidines and a glutamic acid (TroA) [33] whereas the $\mathrm{Zn}$-specific PBPs coordinate the metal by three conserved histidines and by a glutamic acid or a water molecule in the fourth coordination position. The sequence alignment with the other PBPs brought out the presence of a loop between the two secondary structure segments, $\alpha 3 \mathrm{c}$ and $\alpha 4$, in the proteins belonging to the ZnuA family. This loop, absent in the other PBS proteins, is a structural feature necessary for zinc recruitment in the periplasm (see below).

\section{Salmonella enterica ZnuA x-ray structures}

The x-ray crystal structures of recombinant $S$. enterica ZnuA purified from E. coli cells overexpressing the protein [16] were solved in two different forms: ZnuA with a partially occupied zinc-binding site (occupancy $=0.7$ ) solved at $1.7 \AA$ resolution (SeZnuA) (PDB code $2 \mathrm{XY} 4$ ) and $\mathrm{ZnuA}$ with a fully occupied $\mathrm{Zn}$ (II)-binding site ( $\mathrm{Zn}(\mathrm{II})-S e \mathrm{ZnuA})$ solved at $1.9 \AA$ resolution (PDB code 2XQV). The two forms share the same overall fold except for the short 57-61 region which participates in $\mathrm{Zn}$ (II) coordination and assumes a different conformation in the two structures. Accordingly, the superposition of the $\mathrm{C} \alpha$ traces of $\mathrm{SeZnuA}$ and $\mathrm{Zn}(\mathrm{II})-\mathrm{SeZnuA}$ yields a root-mean-square deviation (rmsd) of $0.356 \AA$ [34]. As the other ZnuA proteins whose structures were solved so far, namely Synechocystis ZnuA (SynZnuA, PDB code 1PQ4) and E. coli ZnuA (EcZnuA, PDB codes 2OSV and 2OGW), SeZnuA is constructed by two $(\alpha / \beta)_{4}$ sandwich domains related by pseudo twofold symmetry (comprising residues 27-173 and residues 204-313, respectively) connected by a long $\alpha$-helix (residues $174-$ 203) (Figure $1 \mathrm{~A}$ ). In the $\mathrm{ZnuA} x$-ray structures solved, residues 118-139 belonging to the long His-rich loop

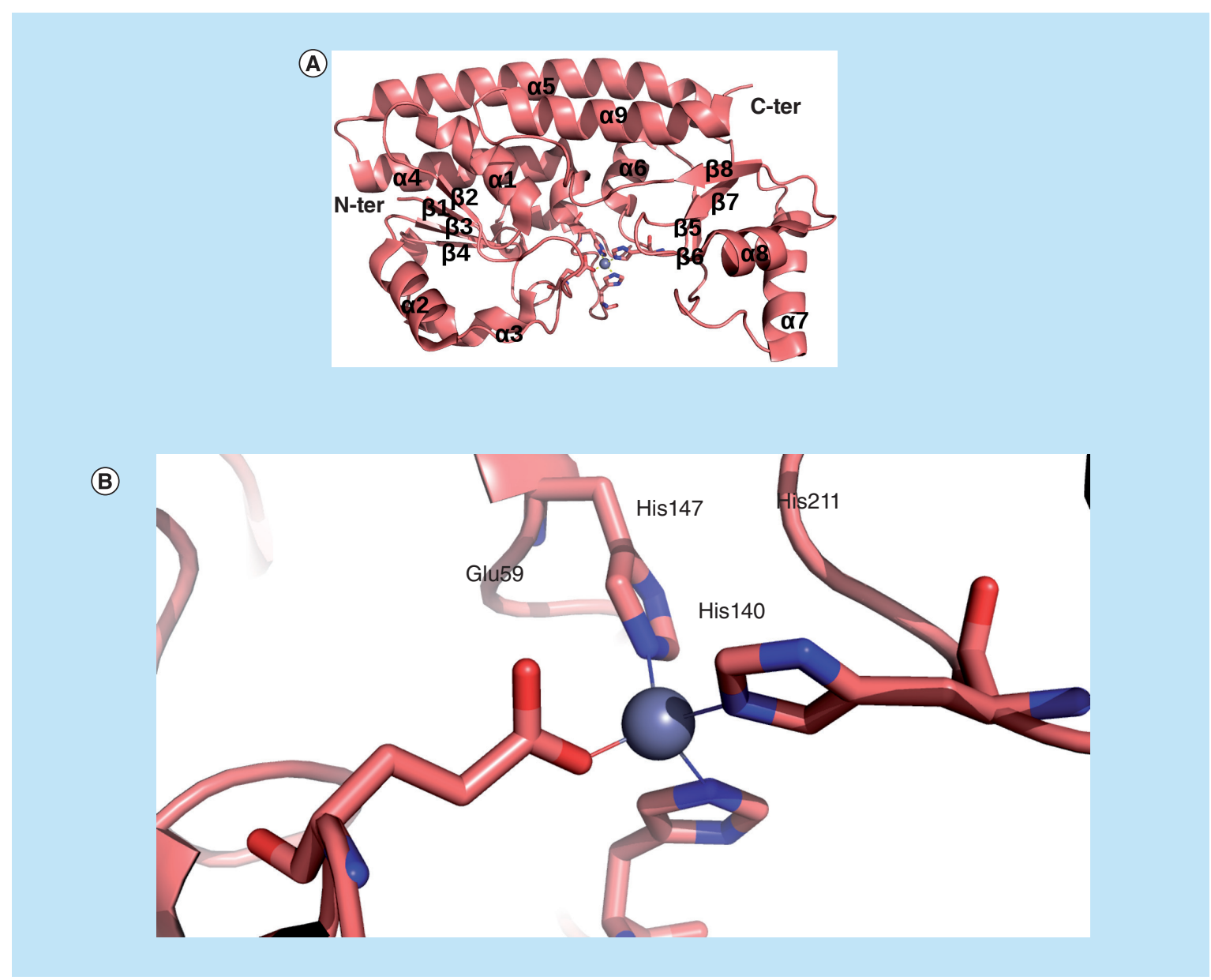


(C)

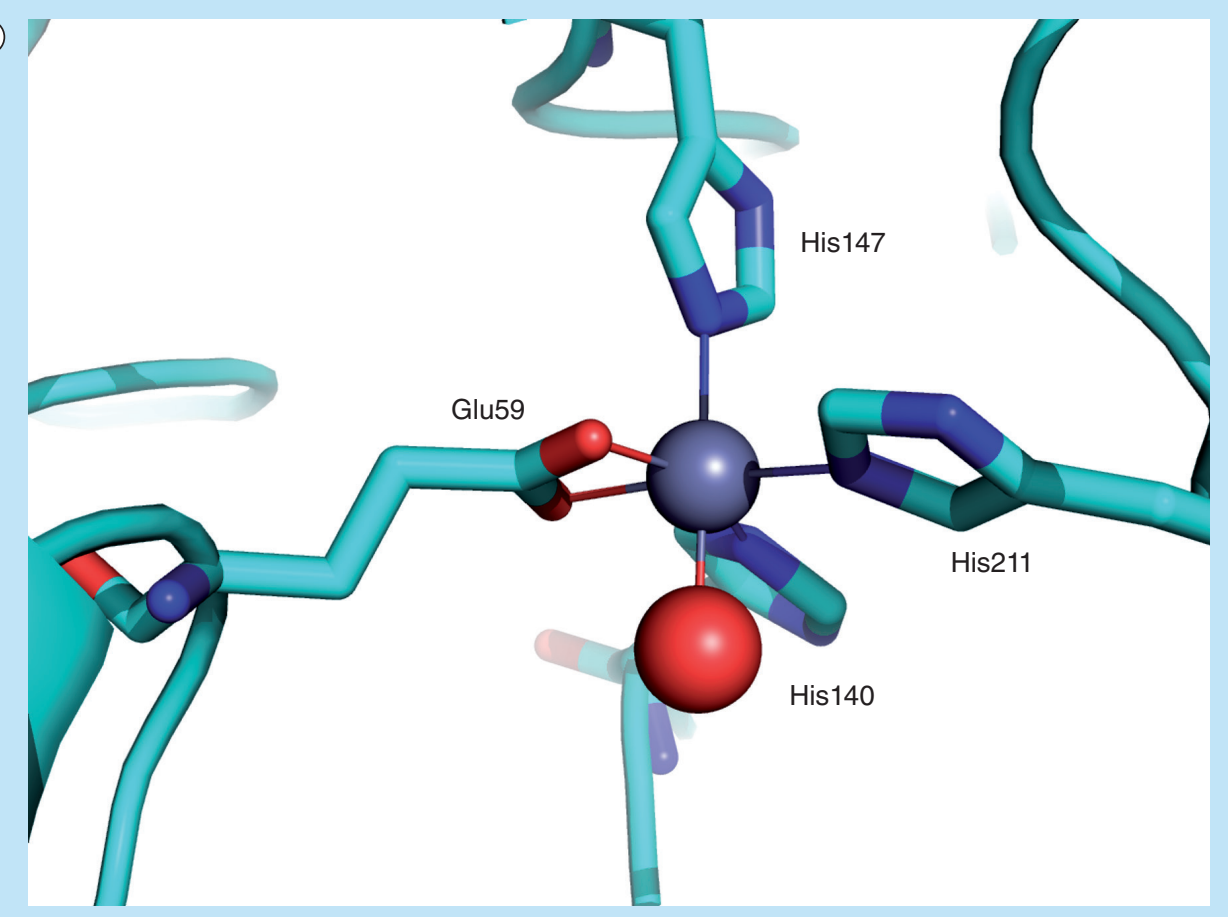

(D)

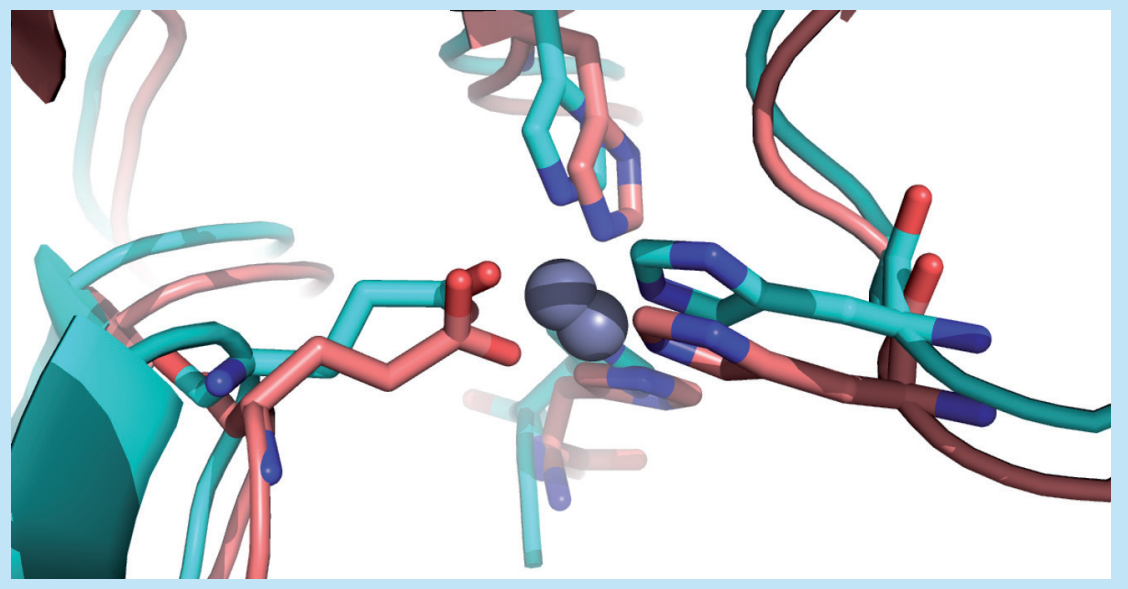

Figure 1. SeZnuA structure (cont. from previous page) (A) Overall fold of the Zn(II)-SeZnuA. The secondary structure elements are are indicated. (B) Zinc-binding site in the $x$-ray structure of Zn (II)-SeZnuA (PDB code 2XQV). The residues binding $\mathrm{Zn}(\mathrm{II})$ are depicted as sticks. $\mathrm{Zn}$ ion is depicted as a grey sphere. (C) Partially occupied zinc-binding site in SeZnuA (PDB code $2 X Y 4$ ). The residues binding $\mathrm{Zn}$ (II) are depicted as sticks. The water molecule is depicted as red sphere. $\mathrm{Zn}$ ion is depicted as a grey sphere. (D) Superimposition between the zincbinding site of Zn(II)-SeZnuA (pink) and SeZnuA (lime-green) (partially occupied by zinc). The pictures have been generated using PyMol [35] and Gimp [36].

between the secondary structural elements $\alpha 3 \mathrm{c}$ and $\alpha 4$ are not visible in the structure. Quite unexpectedly, in SeZnuA one of the histidines of the His-rich loop namely His140 coordinates zinc. In both Salmonella structures solved, the primary $\mathrm{Zn}(\mathrm{II})$-binding site was identified by a strong peak in an anomalous Fourier map in a position shifted by about $1.0 \AA$ from the metal-binding site in $E c$ ZnuA and by about $1.5 \AA$ from that in SynZnuA.
In $\mathrm{Zn}(\mathrm{II})-S e \mathrm{ZnuA}$, the zinc-binding site is fully occupied and is coordinated tetrahedrally by the His147 and His 211 residues, highly conserved among the PBS family members (NE2-Zn(II) distances of 2.2 and $2.0 \AA$, respectively), by Glu59 (OE1-Zn(II) distance $=1.7 \AA$ ) and by His140 (NE2-Zn(II) distance $=2.1 \AA$ ) belonging to the long His-rich loop (Figure 1B). In SeZnuA, where the metal-binding site is partially occupied (occupancy 


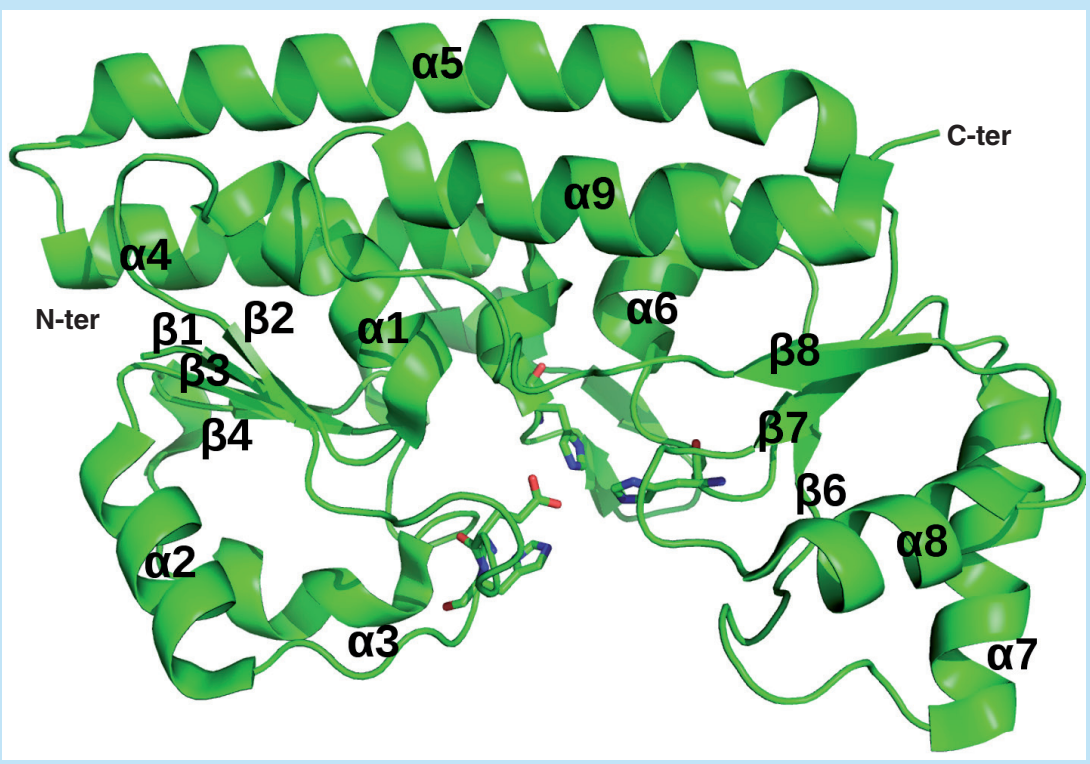

Figure 2. Overall fold of the ZnuA $\Delta \mathbf{1 1 8}-\mathbf{1 4 1}$ deletion mutant. The residues coordinating zinc and the secondary structure elements are indicated.

$=0.7$ ), the map of the 57-61 region is not well defined indicating a higher mobility with respect to the overall structure where the B factor is lower $\left(45 \AA^{2}\right.$ vs $\left.27 \AA^{2}\right)$. The 57-61 region coordinates $\mathrm{Zn}(\mathrm{II})$ in a conformation different from that adopted in the $\mathrm{Zn}-\mathrm{SeZnuA}$ structure, in other words, the side chains of Ser58 and Asp61 appear to be rotated by approximately $180^{\circ}$ and that of His 60 by approximately $90^{\circ}$ around the $C \beta$ carbon with respect to the position adopted in $Z n-S e Z n u A$. As a result, the metal is not tetracoordinated as in $\mathrm{Zn}(\mathrm{II})-S e \mathrm{ZnuA}$, but is hexacoordinated, with a water molecule as sixth ligand $(\mathrm{HOH}-\mathrm{Zn}(\mathrm{II})=2.1 \AA$ ) (Figure 1C, 1D). Figure 1. SeZnuA structure. (A) Overall fold of the Zn(II)-SeZnuA. The secondary structure elements are are indicated. (B) Zinc-binding site in the $x$-ray structure of Zn (II)-SeZnuA (PDB code $2 \mathrm{XQV}$ ). The residues binding $\mathrm{Zn}(\mathrm{II})$ are depicted as sticks. $\mathrm{Zn}$ ion is depicted as a grey sphere. (C) Partially occupied zinc-binding site in SeZnuA (PDB code $2 X Y 4$ ). The residues binding $\mathrm{Zn}$ (II) are depicted as sticks. The water molecule is depicted as red sphere. $\mathrm{Zn}$ ion is depicted as a grey sphere. (D) Superimposition between the zinc-binding site of Zn(II)-SeZnuA (pink) and SeZnuA (lime-green; partially occupied by zinc). The pictures have been generated using PyMol [35] and Gimp [36].

\section{Structure of the His-rich loop deletion mutant SeZnuA $\Delta$ 118-141}

In order to understand the role of the His-rich loop in metal recruitment and coordination, the structure of a SeZnuA $\Delta 118-141$ deletion mutant (SeZnuA $\Delta 118-141$ ) was solved at $2.0 \AA$ resolution (PDB code
2XH8). As expected, in view of the mobility of the deleted region, the structure is identical to the wildtype one. Indeed, the superposition of the SeZnuA and SeZnuA $\Delta 118-141 \mathrm{C} \alpha$ traces yields an rmsd of $0.273 \AA$ indicating that the deletion does not cause a conformational change affecting the protein's overall architecture. The only remarkable structural changes are located on the protein portion belonging to Hisrich loop that has not been deleted (residues 114-117 and 142-147). Indeed, the two segments 114-117 and 142-147 pair to form an additional two-strand antiparallel $\beta$-sheet (Figure 2) [34]. Interestingly, removal of the long His-rich loop and binding of $\mathrm{Zn}$ (II) have the same stabilizing effect on the 57-61 region as clearly shown by the mean B factor values of this part of the structure which upon His-loop removal or $\mathrm{Zn}$ (II) binding become similar to the overall ones in the vari-

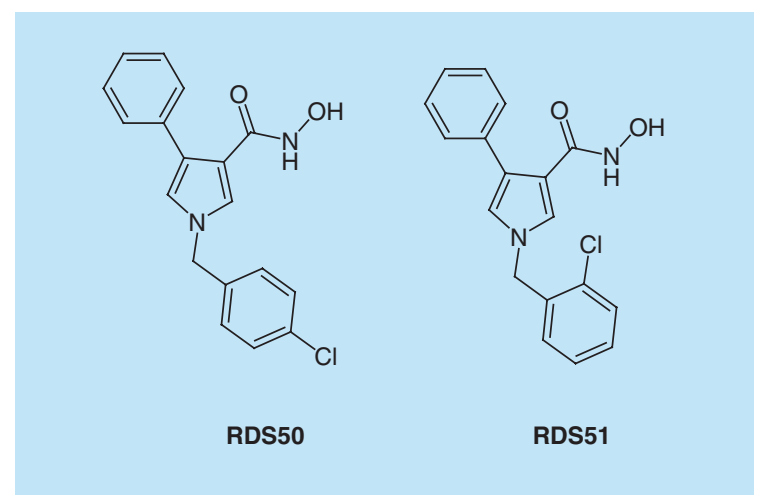

Figure 3. Structural formulas of RDS50 and RDS51. 


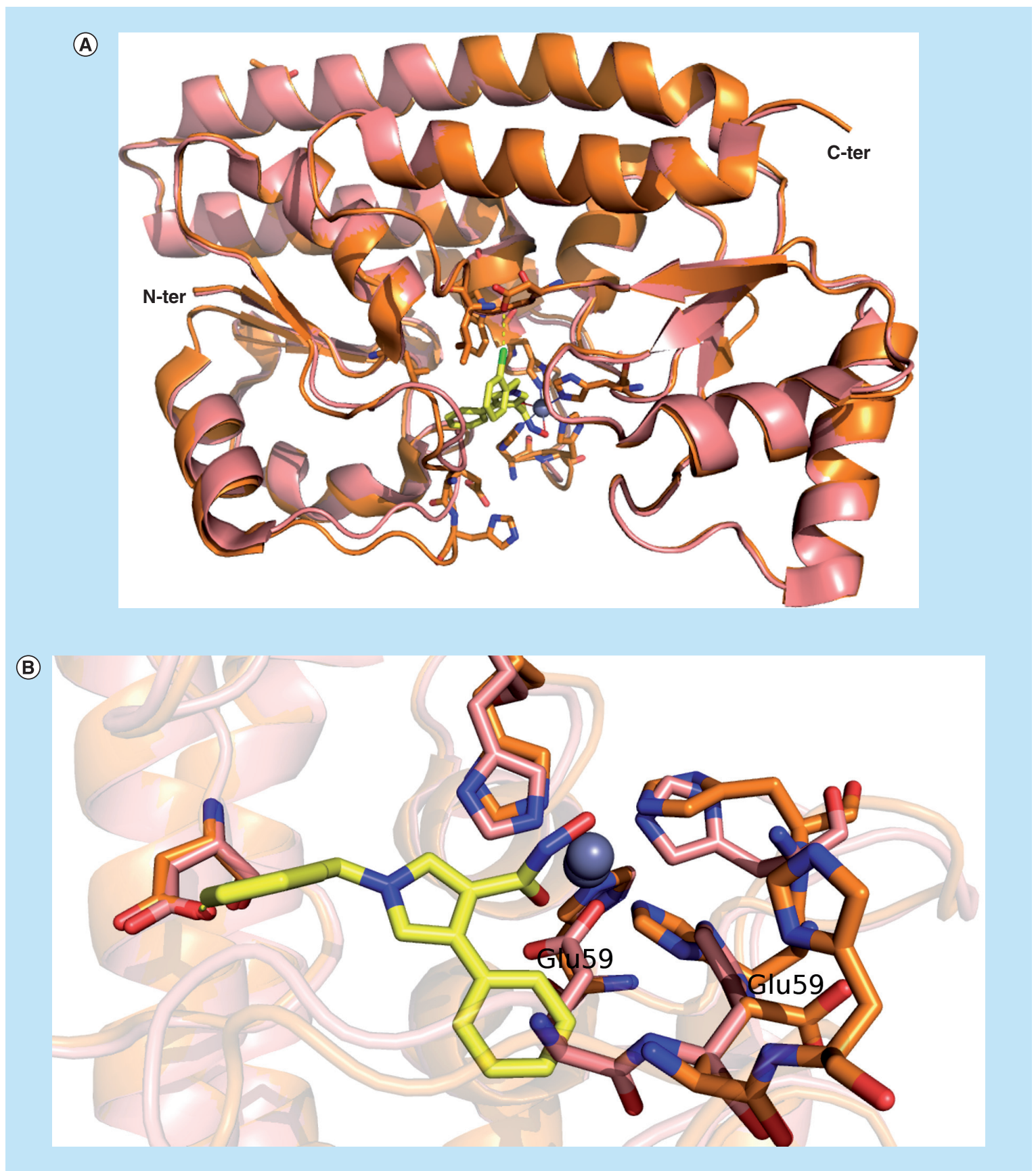

Figure 4. Complex between ZnuA and RDS51. (A) Superimposition between the Zn(II) RDS51-ZnuA complex (in orange) (PDB code 4BBP) and Zn(II)-ZnuA (in salmon) (PDB code 2XQV) (B) Blow-up of the superimposed zincbinding sites. The residues coordinating the zinc ion, Glu59 and His60 of both structures are depicted as sticks. The Glu59 residues of both structures are indicated. (C) Blow-up of the RDS51 binding site. The residues coordinating zinc and the residues lining the hydrophobic pocket accommodating the phenyl ring of RDS51 are indicated.

ous structures analyzed. Thus, while in SeZnuA, the overall B factors and the B factor of the 57-61 loop are quite different (27 $\AA^{2}$ and $45 \AA^{2}$, respectively); in the $\Delta 118-141$ deletion mutant and in $\mathrm{Zn}(\mathrm{II})-\mathrm{SeZnuA}$, the overall $\mathrm{B}$ factors and those of the 57-61 region are quite similar, namely $33.8 \AA^{2}$ and $30 \AA$ versus 35 $\AA^{2}$ and $34 \AA^{2}$, respectively. It is of interest that the attempts to obtain $\mathrm{x}$-ray quality crystals of $\mathrm{Zn}(\mathrm{II})$ bound SeZnuA $\Delta 118-141$ by co-crystallization or in soaking experiments on preformed crystals had no success, indicating that the His-rich loop is a structural feature fundamental for zinc recruitment.

\section{Inhibition of ZnuA activity}


Knowledge of the different SeZnuA structures offers the unique opportunity to design new inhibitors able to impair zinc transport in S. enterica. With this purpose, Ilari et al. [37] screened a small library of compounds chosen from the in-house library of Roberto Di Santo at Sapienza University of Rome, on the basis of their ability to bind zinc. Only two of them: RDS50 [1-[(4-chlorophenyl)methyl]-4-phenyl-1 H-pyrrol3-hydroxamic acid] and RDS51 [1-[(2-chlorophenyl) methyl]-4-phenyl-1 H-pyrrol-3-hydroxamic acid] (Figure 3) belonging to the pyrrolyl-hydroxamate family showed an inhibition activity on wild-type $S$. Typhimurium growth at a concentration of $250 \mu \mathrm{M}$ in Vogel-Bonner minimal medium. The activity of the two compounds improves when they are saturated with $\mathrm{Zn}(\mathrm{II})$. In particular, the $\mathrm{IC}_{50}$ value for $\mathrm{Zn}(\mathrm{II})$ saturated RDS51 and (Zn)-free RDS51 in minimal medium was $221 \mu \mathrm{M}$ and $128 \mu \mathrm{M}$, respectively. Moreover, RDS51 is not toxic for human cells, thereby ruling out the possibility that this small inhibitor may efficiently target zinc-binding proteins of the host organism, and decreases the ability of Salmonella to invade human host cells (Caco-2 cells) in both the apo and zinc saturated form [37].

\section{The x-ray structure of Se-ZnuA in complex with RDS51, an inhibitor molecule}

In order to understand the molecular basis of the pyrrolyl-hydroxamate antibacterial action, the $\mathrm{x}$-ray structure of the complex between RDS51 and recombinant $\operatorname{SeZnuA}$ was solved at $2.15 \AA$ resolution (PDB code $4 \mathrm{BBP})$. The structure showed that RDS51 in complex with $\mathrm{Zn}$ (II) binds to the cavity between the two $(\alpha / \beta)_{4}$ domains of the protein at the zinc-binding site (Figure 4A). Binding of the compound does not modify the overall fold as indicated by the superimpositions of the RDS51-SeZnuA complex with the other SeZnuA structures present in the PDB data bank (SeZnuA with the $\mathrm{Zn}$ (II)-binding site occupied either partially or fully by the metal: PDB codes $2 X Y 4$ and 2XQV and SeZnuA $\Delta 118-141$ deletion mutant: PDB code $2 \mathrm{XH} 8$ ) (Figure $4 \mathrm{~A}$ ). Thus, the yield rmsds are in a range between 0.111 and $0.35 \AA$ for the whole protein length, indicating that binding of the compound does not modify the overall fold. The only difference concerns residues 120-136 belonging to the His-rich loop, that are not visible in the complex, and the 51-65 loop, that upon RDS51 binding assumes a different conformation (Figure 4B).

As shown by the structural analysis, the $\mathrm{Zn}$ (II) ion holds RDS51 and protein together since the compound is coordinated by both RDS51 atoms and protein residues thereby forming a stable complex which adopts a distorted square-based bi-pyramidal geometry. The vertices of the square base are occupied by the two hydroxamate oxygen atoms and the nitrogen atoms of His 140 and His147 which belong to the His-rich loop, while the two apical ligands are the nitrogen atoms of the canoni-

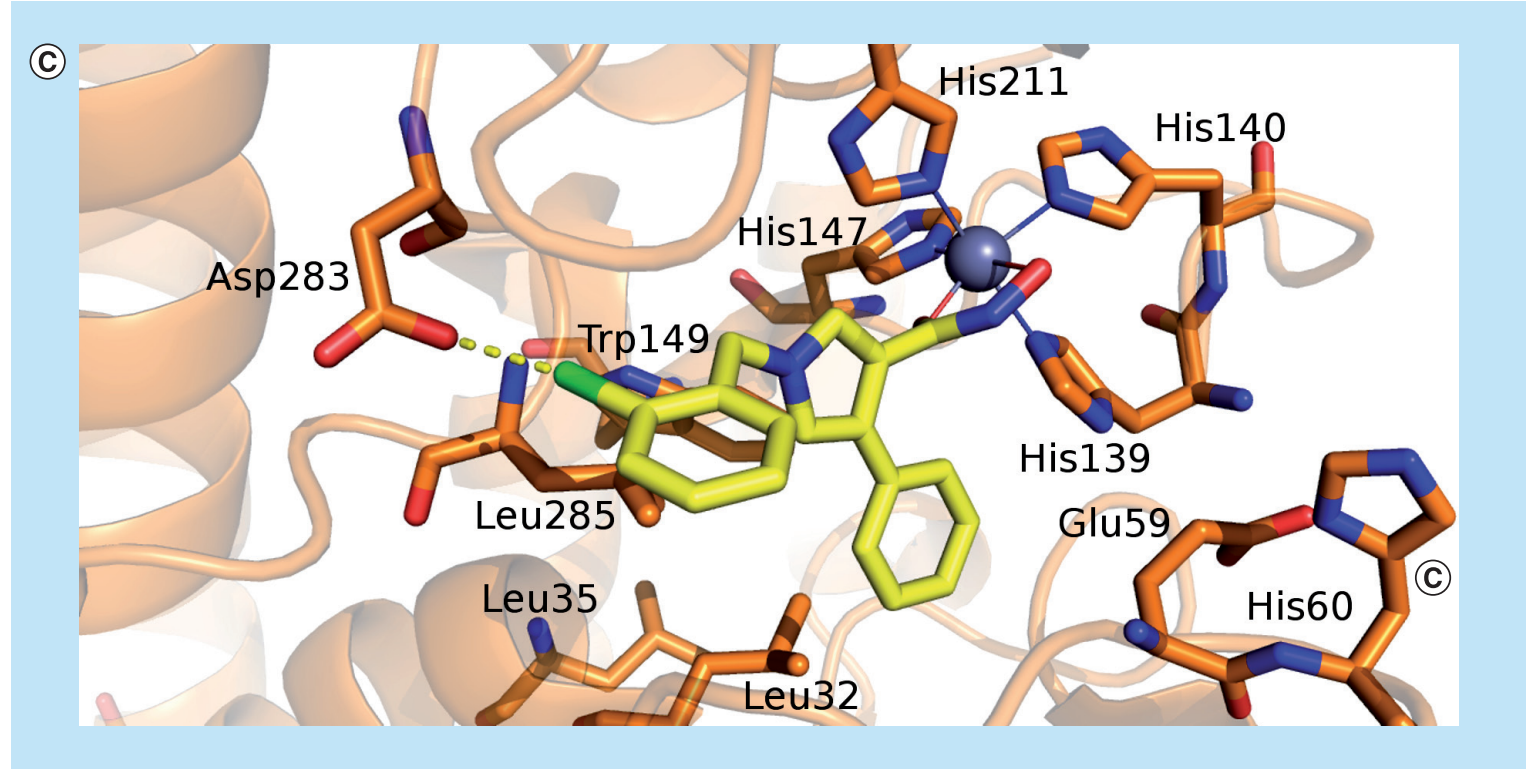

Figure 4. Complex between ZnuA and RDS51 (.Cont. from previous page). (A) Superimposition between the Zn(II) RDS51-ZnuA complex (in orange) (PDB code 4BBP) and Zn(II)-ZnuA (in salmon) (PDB code 2XQV) (B) Blow-up of the superimposed zinc-binding sites. The residues coordinating the zinc ion, Glu59 and His60 of both structures are depicted as sticks. The Glu59 residues of both structures are indicated. (C) Blow-up of the RDS51 binding site. The residues coordinating zinc and the residues lining the hydrophobic pocket accommodating the phenyl ring of RDS51 are indicated. 
SeZnuA

EcZnuA

PaZnuA

SynZnuA

SeZnuA

EcZnuA

PaZnuA

SynZnuA

SeZnuA

EcZnuA

PaZnuA

SynZnuA

SeZnuA

EcZnuA

PaZnuA

SynZnuA

SeZnuA

EcZnuA

PaZnuA

SynZnuA

SeZnuA

EcZnuA

PaZnuA

SynZnuA

MLQK - . - . - - NTLL - - - - -FA - ALSA - . - . - ALWGSATQAADAAVVASLKPLGF

MLHK - - . - - - KTLL - - - - FA - - ALSA - . - - ALWGGATQAADAAVVASLKPVGF

MTLR - . - . - - PFAL - . - - LS - - TCFA - . - . - LFL LASPARAEVSVL TS I KPLQL MF I FPAVPRFVQPLGVAFV LGLSTLGCQPAVEQVGQNGQVEDAPVADAMDI TVS I PPQQY *

I ASA I ADGVTDTQVLLPDGASEHDYSLRPSDVKRLQGADLVVWVGPEMEAFMEKSVRN I P I ASA I ADGVTETE VLL PDGASEHDYSLRPSDVKRLQNADLVVWVGPEMEAFMQKPVSK LP VAAA IQDGVGQPDVLL PPGASPHQYALRPSDVRRLREVQL FYWI GPDLENFLPKVLAGRQ FLEK I GGDLVRVSVLVPGNNDPHTYEPKPQQLAA LSEAEAYVL I GLGFEQPWLEKLKAAN

DNKQVTI AQL ADVKPLLMKGADD - - - - - DEDEHAHTGADEEKG- - - -DVHHHHGEYNMㅂ 147 GAKQVT I AQLEDVKP L LMKS IHG - . . . DDDDHDHA - . - EKS - . - DEDHHHGDFNMH 143 GTS - . - - VAVQDLPGMHLRKFV - . . - . - NFEEEEHAGH - - DEH - . - -DHDHRPGMLDA 140 AN-MKLI DSAQGI TPLEMEKHDHSHGEEEGHDDHSHDGHDHGSESEKEKAKGALMVADP $\underline{\underline{H}} 179$

LWLSPE I ARATAVA I HEKL VELMPQSRAKLDANL KDFEAQLAATDKQVGNELAPL KGKGY 207 LWLSPE I ARATAVA I HGKLVE LMPQSRAKLDANLKDFEAQLAS TETQVGNELAPLKGKGY 203 LWLLPANART I AARMAEDLAQVDPANAGRYRANL KAFDERLGGLDGKLRERLGKLAGKPF 200 I WLSPTL V KRQAT T I AKELAEL DPDNRDQYEANLAAFLAELERLNQELGQI LQPLPQRKF 239

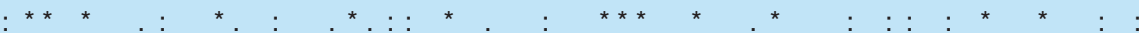

FVFHDAYGYYEKHYGL TPLGHFTVNPE I QPGAQRLHE IRTQLVEQKATCVFA EPQFRPAV 267 FVFḦDAYGYFEKQFGLTP LGHFTVNPEI QPGAQRLHE IRTQLVEQKATCVFAEPQFRPAV 263 FVFHEAF DYFEEA YGLRHTGVFAVSAEVQPGARHVAAMRAQLKAAGPAC I FSEPPLRPRL 260 I VF $:{ }^{* *}::{ }^{*}: .:{ }^{*}:{ }^{*} .{ }^{*}:: \quad: \quad:{ }^{*}{ }^{*}$

VEAVARGTSVRMGTLDPLGTNIKLGKTSYSAFLSQLANQYASCLKGDVESVARGTSVRMGTLDPLGTNIKLGKTSYSEFLSQLANQYASCLKGD-

Figure 5. Sequence alignment of SeZNuA, SynZnuA, PaZnuA and EcZnuA. The sequence alignment was performed with the program Clustal Omega [45]. The His-rich loop is colored red; the loop 51-65 containing His60, one of the conserved zinc ligand and Glu59, which in SeZnuA is one of the zinc coordinating residue, is colored blue. The conserved zinc ligands are in bold and underlined whereas the conserved residues interacting with the $\mathrm{Zn}$ (II)RDS51 complex in SeZnuA are indicated in yellow.

cal His211 residue and His139 also belonging to the Hisrich loop (Figure 4C). The distances between the coordinating ligands and $\mathrm{Zn}(\mathrm{II})$ range between 2.0 and $2.3 \AA$.

Upon RDS51 binding, the protein undergoes a local conformational change that involves only the loops surrounding the active site. Thus, RDS51 binding causes a movement of the entire 55-61 region and in particular of the bidentate $\mathrm{Zn}(\mathrm{II})$ ligand Glu59 which is shifted and rotated with respect to its position in the other ZnuA structures (Figure 4B). One of the most remarkable finding is the disclosure of the role of His 139 which is clearly visible in the complex structure (but not in the other SeZnuA structures) where it is, as described above, one of the $\mathrm{Zn}$ (II) ion ligand. The RDS51 binding causes a conformational change eliciting the opening of the big cavity located at the interface between the two Se-ZnuA subdomains which displays an area of $758.4 \AA^{2}$ and a volume of $1511 \AA^{3}$ and is lined by the following residues:
Leu285, Leu32, Lys33, Leu53, Asp55, Gly56, Ala57, Ser58, Glu59, His60 and Trp149 (Figure 4C).

As shown in Figure 4C, the RDS51 compound interacts with hydrophobic residues lining the cavity. In particular, it forms hydrophobic interactions with the Leu285, Leu32 and Trp149 residues. Moreover, it interacts electrostatically by means of the chloride atom with an aspartic acid located at the entrance of the cavity $(\mathrm{O}-$ Asp283-RDS51 chloride $=2.9 \AA)$ [37]. RDS51 is, to our knowledge, the first proved inhibitor of the ZnuABC transporter and could be a lead compound to find new and more effective molecules able to interfere with the Gram-negative bacteria zinc metabolism.

\section{Conclusion \& future perspective}

The results discussed in this review recapitulate the available evidence that $\mathrm{ZnuABC}$ activity is indispensable to guarantee an adequate zinc import to $S$. enterica and plays 
a critical role in the ability of the pathogen to establish successful infections in the infected host. It follows that it may represent a good target to obtain new drugs decreasing Salmonella virulence and infectivity. Indeed, the identification of the first ZnuA inhibitors (RDS50 and RDS51) which impedes the zinc transport through the inner membrane in the cytoplasm of $S$. Typhimurium thereby inhibiting its growth provides a proof of principle of the feasibility of this approach. In addition, the availability of $\mathrm{x}$-ray structures disclosing the structural features required for the recruitment of zinc in the periplasmic space and revealing the details of the interaction between the protein and $\mathrm{Zn}(\mathrm{II})-\mathrm{RDS} 51$ provides the basis for rational improvements of the inhibitor and for the identification of new molecules able to interfere with ZnuA-mediated zinc import. The observation that ZnuA inhibition is favored by the RDS51-Zn complex suggests that one important characteristic of effective ZnuABC inhibitors should be the ability to bind zinc.

Beyond the demonstration that it is possible to interfere with the growth of a specific microorganism through inhibition of $\mathrm{ZnuABC}$, these studies can be placed within a broader context, namely that interfering with zinc homeostasis may represent a very promising strategy for defeating pathogens. Along this line, based on the observation that fungal pathogens are extremely sensitive to zinc deprivation, Simm et al. successfully screened a library of chemical compounds to identify drugs able to target the transcription factor Zapl which regulates the expression of a high-affinity zinc transporter in Candida albicans [38]. Moreover, very recent studies on the mechanism of action of dithiolopyrrolones, have revealed that the antimicrobial activity of these natural substances is correlated to their ability to chelate intracellular zinc [39].

An important issue related to the present study is to understand whether drugs targeting Salmonella ZnuA may be useful to develop antimicrobials against other Gram-negative pathogens. The sequence alignment of SeZnuA protein with the ZnuA of other Gram-negative bacteria (Figure 5) shows that the residues lining the hydrophobic pocket as well as Aps283 located at the entrance of the pocket are strongly conserved. Thus, RDS51 and in general compounds inhibiting SeZnuA may also inhibit this importer in other pathogenic bacteria. However, whereas in Salmonella and other gastrointestinal Gram-negative pathogens, ZnuABC is the unique high-affinity zinc importer, other bacteria possess redundant zinc importers [9], thereby questioning the general usefulness of drugs targeting ZnuA to treat infections supported by Gram-negative bacteria. For example, Pseudomonas aeruginosa mutant strains lacking the ZnuABC zinc transporter are able to efficiently proliferate in media containing only traces of zinc, likely

\section{Executive summary}

- The discovery of novel antimicrobials is a major challenge to overcome the problem of antibiotic resistance, and significant efforts have been made to identify effective targets for new antimicrobials. Salmonella enterica sv Typhimurium causing self-limiting gastroenteritis in humans is a good model for the identification of antimicrobial targets in Gram-negative bacteria.

- The intracellular supply of zinc is primarily provided in Gram-negative bacteria by the low-affinity importer ZupT, an integral inner membrane protein that belongs to the ZIP (ZRT, IRT-like protein) family and ZnuABC which ensures metal import under conditions of zinc shortage.

- Deletion of the $z n U A B C$ operon does not alter Salmonella ability to grow in zinc-rich media but impairs its ability to grow in environments with low zinc content as the inflamed gut of infected human host.

- Proteomic analyses of Salmonella have evidenced that inactivation of the ZnuABC transporter determines major ionomic and proteomic intracellular changes under zinc shortage conditions. In particular, several virulence-related proteins and proteins necessary for Salmonella motility are downregulated in the znuA mutant, grown under zinc stringency.

- Since ZnuABC plays a central role in Salmonella pathogenicity, this transporter, or merely its soluble periplasmic component ZnuA, represents putative targets to find new drugs against Gram-negative bacteria.

- The x-ray structures of $S$. enterica ZnuA disclose the structural features necessary for zinc recruitment and binding.

- The x-ray structure of the His-rich loop deletion mutant (SeZnuA $\Delta 118-141$ ) furnishes an additional proof that the His-rich loop is a structural feature necessary to bind and release zinc in the periplasmic space.

- RDS50 [1-[(4-chlorophenyl)methyl]-4-phenyl-1 H-pyrrol-3-hydroxamic acid] and RDS51 [1-[(2-chlorophenyl) methyl]-4-phenyl-1 H-pyrrol-3-hydroxamic acid] are the two pyrrolyl-hydroxamate family members that have been identified as the first ZnuA inhibitors.

- The x-ray structure of the complex between Zn(II)-RDS51 and ZnuA discloses the presence of a druggable pocket conserved among the Gram-negative ZnuA proteins.

- The studies presented here shows that ZnuABC inhibition is a promising strategy to find new weapons to combat microbial infections. 
due to the activity of other compensatory zinc uptake systems [40-42]. However, even though the $P$. aeruginosa znuA mutant strain grows in environments poor of zinc, it shows a remarkable reduction in cellular zinc accumulation [42] and displays a significant alteration in the expression of major virulence features [40]. Thus, znuA mutants show: reduced resistance to calprotectin inhibition; decreased secretion of extracellular zinccontaining proteases (LasA, LasB and Protease IV); and lower ability to produce alginate, which is required for the formation of biofilm. Moreover, intracellular variations in zinc concentration could have an impact on the resistance to $\beta$-lactam antibiotics that involves the expression of enzymes involving $\mathrm{Zn}$ (II) ions as essential factors or involves modulation in the expression of the porin $\mathrm{OprD}$, a protein known to regulate

\section{References}

Papers of special note have been highlighted as: $\bullet$ of interest;

•• of considerable interest

1 Spellberg B, Shlaes D. Prioritized current unmet needs for antibacterial therapies. Clin. Pharmacol. Ther. 96(2), 151-153 (2014).

2 Coates AR, Halls G, Hu Y. Novel classes of antibiotics or more of the same? BJP 163(1), 184-194 (2011).

3 Jelsbak L, Hartman $\mathrm{H}$ et al. Identification of metabolic pathways essential for fitness of Salmonella Typhimuriumin vivo. PLoS ONE 9(7), e101869 (2014).

4 Chaudhuri RR, Peters SE et al. Comprehensive identification of Salmonella enterica serovar Typhimurium genes required for infection of BALB/c mice. PLoS Pathog. 5(7), e1000529 (2009).

5 Becker D, Selbach M et al. Robust Salmonella metabolism limits possibilities for new antimicrobials. Nature 440 (7082), 303-307 (2006).

6 Mislin GL, Schalk IJ. Siderophore-dependent iron uptake systems as gates for antibiotic Trojan horse strategies against Pseudomonas aeruginosa. Metallomics 6(3), 408-420 (2014).

7 Gorska A, Sloderbach A, Marszall MP. Siderophore-drug complexes: potential medicinal applications of the 'Trojan horse' strategy. Trends Pharmacol. Sci. 35(9), 442-449 (2014).

8 Bonchi C, Imperi F, Minandri F, Visca P, Frangipani E. Repurposing of gallium-based drugs for antibacterial therapy. Biofactors 40 (3), 303-312 (2014).

9 Cerasi M, Ammendola S, Battistoni A. Competition for zinc binding in the host-pathogen interaction. Front. Cell. Infect. Microbial. 3, 108 (2013).

10 Delcour AH. Outer membrane permeability and antibiotic resistance. Biochim. Biophys. Acta 1794(5), 808-816 (2009).

11 Grass G, Wong MD, Rosen BP et al. ZupT is a Zn(II) uptake system in Escherichia coli. J. Bacteriol. 184(3), 864-866 (2002).

12 Grass G, Franke S, Taudte N et al. The metal permease ZupT from Escherichia coli is a transporter with a broad substrate spectrum. J. Bacteriol. 187(5), 1604-1611 (2005). resistance to carbapenems [43,44]. Taken together, these observations suggest that the search for compounds capable of blocking the import of zinc mediated by ZnuABC or other high-affinity metal transporters is a promising strategy to find new weapons to combat microbial infections.

\section{Financial \& competing interests disclosure}

The authors have no relevant affiliations or financial involvement with any organization or entity with a financial interest in or financial conflict with the subject matter or materials discussed in the manuscript. This includes employment, consultancies, honoraria, stock ownership or options, expert testimony, grants or patents received or pending, or royalties.

No writing assistance was utilized in the production of this manuscript.

13 Cerasi M, Liu JZ, Ammendola S et al. The ZupT transporter plays an important role in zinc homeostasis and contributes to Salmonella enterica virulence. Metallomics 6(4), 845-853 (2014).

14 Karlinsey JE, Maguire ME, Becker LA, Crouch ML,Fang FC. The phage shock protein PspA facilitates divalent metal transport and is required for virulence of Salmonella enterica sv. Typhimurium. Mol. Microbiol. 78(3), 669-685 (2010).

15 Hantke K. Bacterial zinc uptake and regulators. Curr. Opin. Microbiol. 8(2), 196-202 (2005).

- This is an interesting review which describes the mechanisms of zinc transport in bacteria and their regulation.

16 Petrarca P, Ammendola S, Pasquali P, Battistoni A. The Zur-regulated ZinT protein is an auxiliary component of the high-affinity ZnuABC zinc transporter that facilitates metal recruitment during severe zinc shortage. J. Bacteriol. 192(6), 1553-1564 (2010).

17 Ilari A, Alaleona F, Tria G et al. The Salmonella enterica ZinT structure, zinc affinity and interaction with the high-affinity uptake protein ZnuA provide insight into the management of periplasmic zinc. Biochim. Biophys. Acta 1840(1), 535-544 (2014).

18 Gilston BA, Wang S, Marcus MD et al. Structural and mechanistic basis of zinc regulation across the $E$. coli Zur regulon. PLoS Biol. 12(11), e1001987 (2014).

19 Shin JH, Helmann JD. Molecular logic of the Zur-regulated zinc deprivation response in Bacillus subtilis. Nat. Commun. 7, 12612 (2016).

20 Ammendola S, Pasquali P, Pistoia C et al. High-affinity $\mathrm{Zn}^{2+}$ uptake system $\mathrm{ZnuABC}$ is required for bacterial zinc homeostasis in intracellular environments and contributes to the virulence of Salmonella enterica. Infect Immun. 75 , 5867-5876 (2007).

- Shows that the amount of free metals available for bacterial growth within the infected host is limited and suggests that during the infection Salmonella exploits the ZnuABC zinc transporter to maximize zinc recruitment.

21 Liu JZ, Jellbauer S, Poe AJ et al. Zinc sequestration by the neutrophil protein calprotectin enhances Salmonella growth 
in the inflamed gut. Cell Host Microbe 11(3), 227-239 (2012).

- Shows that ZnuABC is essential for Salmonella growth in the inflamed gut since it mediates resistance to neutrophil antimicrobial strategy.

22 Drumo R, Pesciaroli M, Ruggeri J et al. Salmonella enterica serva Typhimurium exploits inflammation to modify swine intestinal microbiota. Front. Cell. Infect. Microbiol. 5, 106 (2016).

23 Ciavardelli D, Ammendola S, Ronci M et al. Phenotypic profile linked to inhibition of the major $\mathrm{Zn}$ influx system in Salmonella enterica: proteomics and ionomics investigations. Mol. Biosyst. 7(3), 608-619 (2011).

24 Ammendola S, Cerasi M, Battistoni A. Deregulation of transition metals homeostasis is a key feature of cadmium toxicity in Salmonella. Biometals 27(4), 703-714 (2014).

25 Gabriel SE, Helmann JD. Contributions of Zur-controlled ribosomal proteins to growth under zinc starvation conditions. J. Bacteriol. 191(19), 6116-6122 (2009).

26 Ammendola S, D'Amico Y, Chirullo B et al. Zinc is required to ensure the expression of flagella and the ability to form biofilms in Salmonella enterica sv Typhimurium. Metallomics 8(10), 1131-1140 (2016).

27 Pasquali P, Ammendola S, Pistoia C et al. Attenuated Salmonella enterica serovar Typhimurium lacking the ZnuABC transporter confers immune-based protection against challenge infections in mice. Vaccine 26(27-28), 3421-3426 (2008).

28 Pesciaroli M, Aloisio F, Ammendola et al. An attenuated Salmonella enterica serovar Typhimurium strain lacking the $\mathrm{ZnuABC}$ transporter induces protection in a mouse intestinal model of Salmonella infection. Vaccine 29(9), 1783-1790 (2011).

29 Gradassi M, Pesciaroli M, Martinelli N et al. Attenuated Salmonella enterica serovar Typhimurium lacking the ZnuABC transporter: an efficacious orally-administered mucosal vaccine against salmonellosis in pigs. Vaccine 31(36), 3695-3701 (2013).

30 Ruggeri J, Pesciaroli M, Gaetarelli B et al. Parenteral administration of attenuated Salmonella Typhimurium $\triangle z n u A B C$ is protective against salmonellosis in piglets. Vaccine 32(32), 4032-4038 (2014).

31 Rukhman V, Anati R, Melamed-Frank M, Adir N. The $\mathrm{MntC}$ crystal structure suggests that import of $\mathrm{Mn}^{+}$in cyanobacteria is redox controlled. J. Mol. Biol. 348(4), 961-969 (2005).

32 Lawrence MC, Pilling PA, Epa VC et al. The crystal structure of pneumococcal surface antigen PsaA reveals a metal-binding site and a novel structure for a putative ABCtype binding protein. Structure 6(12), 1553-1561 (1998).

33 Lee YH, Deka RK, Norgard MV, Radolf JD, Hasemann CA. Treponema pallidum TroA is a periplasmic zinc-binding protein with a helical backbone. Nature Struct. Biol. 6(7), 628-633 (1999).

34 Ilari A, Alaleona F, Petrarca P, Battistoni A, Chiancone E. The X-ray structure of the zinc transporter ZnuA from Salmonella enterica discloses a unique triad of zinccoordinating histidines. J. Mol. Biol. 409(4), 630-641 (2011).

-. The structures of ZnuA from Salmonella enterica demonstrate that the long His-rich loop is a structural feature necessary for zinc recruitment in periplasm.

35 Schrodinger. PyMOL Molecular Graphics System, Version 1.7. http//www.pymol.org

36 The GIMP team. GIMP 2.8.10. www.gimp.org

37 Ilari A, Pescatori L, Di Santo R et al. Salmonella enterica serovar Typhimurium growth is inhibited by the concomitant binding of $\mathrm{Zn}$ (II) and a pyrrolyl-hydroxamate to ZnuA, the soluble component of the ZnuABC transporter. Biochim. Biophys. Acta 1860 (3), 534-541 (2016).

-. In this paper the structure of $\mathrm{ZnuA}$ in complex with RDS51 is analysed. The paper demonstrates that ZnuA from $S$. enterica is druggable.

38 Simm C, Luan CH, Weiss E, O'Halloran T. Highthroughput screen for identifying small molecules that target fungal zinc homeostasis. PLoS ONE 6(9), e25136 (2011).

39 Chan AN, Shiver AL, Wever WJ, Razvi SZ, Traxler MF, Li B. Role for dithiolopyrrolones in disrupting bacterial metal homeostasis. Proc. Natl Acad. Sci. USA doi:10.1073/ pnas.1612810114 (2017) (Epub ahead of print).

40 D'Orazio M. The capability of Pseudomonas aeruginosa to recruit zinc under conditions of limited metal availability is affected by inactivation of the $\mathrm{ZnuABC}$ transporter. Metallomics 7(6), 1023-1035 (2015).

41 Ellison ML, Parrish W, Danell AS, Pesci EC. The transcriptional regulator $\mathrm{Np} 20$ is the zinc uptake regulator in Pseudomonas aeruginosa. PLoS ONE 8(9), e75389 (2013)

42 Pederick VG. ZnuA and zinc homeostasis in Pseudomonas aeruginosa. Sci. Rep. 5, 13139 (2015).

43 Marguerettaz M, Dieppois G, Que YA et al. Sputum containing zinc enhances carbapenem resistance, biofilm formation and virulence of Pseudomonas aeruginosa. Microb. Pathog. 77, 36-41 (2014).

44 Fajardo A, Hernando-Amado S, Oliver A, Ball G, Filloux A, Martinez JL. Characterization of a novel $\mathrm{Zn}^{2+}$-dependent intrinsic imipenemase from Pseudomonas aeruginosa. J. Antimicrob. Chemother. 69(11), 2972-2978 (2014).

45 EMBL-EBI. Clustal Omega. www.ebi.ac.uk 